\title{
The PCI Interface for GRAPE Systems: PCI-HIB
}

\author{
Atsushi Kawai ${ }^{1}$, Toshiyuki Fukushige ${ }^{1}$, Makoto Taiji ${ }^{2}$, \\ Junichiro Makino ${ }^{1}$ and Daiichiro Sugimoto ${ }^{1}$ \\ ${ }^{1}$ Department of General Systems Studies, College of Arts and Sciences, University of Tokyo, \\ 3-8-1 Komaba, Meguro-ku, Tokyo 153 \\ ${ }^{2}$ Institute of Statistical Mathematics, \\ 4-6-7 Azabu, Minato-ku, Tokyo 106
}

\begin{abstract}
We developed a PCI interface for GRAPE systems. GRAPE(GRAvity piPE) is a special-purpose computer for gravitational $N$-body simulations. A GRAPE system consists of GRAPE processor boards and a host computer. GRAPE processors perform the calculation of gravitational forces between particles. The host computer performs the rest of calculations. The newest of GRAPE machines, the GRAPE-4, achieved the peak performance of 1.08 Tflops. The GRAPE-4 system uses TURBOChannel for the interface to the host, which limits the selection of the host computer. The TURBOChannel bus is not supported by any of recent workstations. We developed a new host interface board which adopts the PCI bus instead of the TURBOChannel. PCI is an I/O bus standard developed by Intel. It has fairly high peak transfer speed, and is available on wide range of computers, from PCs to supercomputers. Thus, the new interface allows us to connect GRAPE-4 to a wide variety of host computers. In test runs with a Barnes-Hut treecode, we found that the performance of new system with PCI interface is $40 \%$ better than that of the original system.
\end{abstract}

Subject headings: Clusters: globular — Numerical methods — Stars: stellar dynamics 


\section{Introduction}

Many astronomical objects can be well approximated by gravitational $N$-body systems. To understand the behavior of gravitational $N$-body systems is one of the most important problems in theoretical astrophysics.

Numerical simulation is widely used to study the behavior of $N$-body systems, because in many cases the analytic approach or more idealized method are not sufficient. However, in many fields the limitation in the resolution and accuracy makes it difficult to obtain meaningful results from $N$-body simulations. These limitations come mainly from limited number of particles.

In $N$-body simulations, the calculation speed of the computer is the primary cause to limit the number of particles, since a naive algorithm requires $O\left(N^{2}\right)$ calculation cost per timestep, where $N$ is the number of particles. The calculation cost is $O\left(N^{2}\right)$ because gravity is a long-range interaction. Each particle interacts with all other particles.

In some cases, approximate algorithms such as the $\mathrm{P}^{3} \mathrm{M}$ algorithm and the Barnes-Hut treecode (Barnes and Hut 1986) can be used, resulting in the reduction of calculation cost from $O\left(N^{2}\right)$ to $O(N \log N)$. Even with these schemes, the cost of the gravitational interaction is the dominant part of the total calculation cost. There are a number of studies to improve the efficiency of these schemes, in particular on parallel computers.

We have been exploring an alternative approach, which is to develop a special-purpose hardware for the force calculation. The calculation of the force between two particles is simple enough to be put on a hardwired pipeline (Sugimoto et al. 1990). This hardware works in cooperation with a general-purpose computer (host computer), which does everything except for the force calculation. In the case of the direct force calculation, calculation cost of the force is $O\left(N^{2}\right)$ and that of the rest is $O(N)$. Therefore the requirement for the speed of the calculation on the host and the data transfer between the host and the special-purpose hardware is rather modest. In addition, fast, approximate schemes such as the $\mathrm{P}^{3} \mathrm{M}$ and Barnes-Hut treecode can be further accelerated with the hardware for the particle-particle force (Makino 1991a, Brieu et al. 1994), though in this case the requirement for the performance of the host becomes somewhat higher simply because the total calculation cost is smaller than that for the direct summation.

The GRAPE-4 system (Taiji et al. 1996, Makino et al. 1997) is our newest hardware with the theoretical peak speed of 1.08 Tflops. The measured best speed so far was 523 Gflops, for the simulation of black hole binaries in the center of an elliptical galaxy (Makino and Taiji 1995).

In this paper, we describe the enhancement we added to GRAPE-4 to further improve its performance on real problems. In many simulations, the performance of GRAPE- 4 was limited by the speed of the host computer. This is essentially because GRAPE-4 is so fast, but partly because we could not use the fastest host computer available. The hardware interface to the host is designed around TURBOChannel, the I/O bus specification developed by DEC. At the time of the development of GRAPE-4, it was a reasonable choice, but in 1994 DEC dropped the product line with TURBOChannel.

In order to improve the performance of the host computer, we designed a new host interface for GRAPE4. We adopted the PCI interface as the host interface. PCI (Peripheral Component Interconnect, PCI Special Interest Group 1993) is an I/O bus standard developed by Intel. It is currently the most widely used I/O bus for PCs with Intel x 86 processors, and a number of computer manufacturers of all kinds of products, including vector supercomputers, massively parallel computers, SMP servers and workstations, have shifted from proprietary I/O bus to this PCI bus. Unlike the TURBOChannel, PCI will be around for next 5-10 years, if we can judge its lifetime from that of its predecessor, the ISA bus.

As of 1996, virtually all manufacturers of PC are providing PCI, and all major workstation vendors either are shipping PCI products (DEC, HP, SGI, IBM) or have announced products (SUN). In addition, Apple has also shifted from NuBus to PCI in 1994. Recently announced NEC SX-4A vector supercomputer also supports PCI as I/O interface.

There are several reasons why the PCI bus is now supported on almost all computers. The first one is the production cost. PCI is first adopted by PC manufacturers, which are now producing more than $90 \%$ of the total number of computers. Thus, the peripherals such as display cards, SCSI interface cards and network interface cards with PCI interface are produced in the quantity more than 10 times larger than the total of all other kinds of peripherals, and 
therefore the production cost of the PCI-based interface is much lower. By adapting the PCI bus, any computer can use the peripherals designed for PCs, thereby greatly reducing the total development cost.

The second reason is that the performance of the PCI interface is quite high. Even with the low-end specification, it offers the maximum data transfer rate of $133 \mathrm{MB} / \mathrm{s}$, which is more than enough for almost any storage or communication device. A highperformance hard disk unit would offer the data transfer rate of 10-20 MB/s. Fast Ethernet and ATM network also offer the data transfer rate of $10-15 \mathrm{MB} / \mathrm{s}$. Thus, the speed of $133 \mathrm{MB} / \mathrm{s}$ is more than enough. Even so, high-end PCs and workstations now support $266 \mathrm{MB} / \mathrm{s}$, and the data transfer rate of $533 \mathrm{MB} / \mathrm{s}$ might be implemented soon. This transfer speed is faster than those of other proprietary I/O buses.

The wide variety of computers which support PCI and the high performance of the PCI makes it an ideal choice as the I/O interface for GRAPE hardwares, including the ones we will develop in future. On the other hand, to develop a PCI interface is a challenging task, partly because of its high performance and partly because of its complexity.

In this paper, we describe the implementation and the performance of PCI interface hardware we developed for GRAPE-4. In section 2, we briefly describe the GRAPE-4 system. In section 3, we describe the PCI interface for GRAPE-4. We call it PHIB (PCI host interface board). In section 4, we present the measured performance of PHIB and GRAPE-4 with PHIB. Section 5 is devoted for discussions.

\section{GRAPE-4 System}

In this section, we describe the GRAPE-4 system. In section 2.1, we show the hardware structure of the system. In section 2.2, we describe how the actual $N$-body simulation is performed with this system. In section 2.3, the function of HIB is described.

\subsection{The Hardware Structure}

Figure 1 shows the structure of the GRAPE- 4 system. It consists of a host computer and multiple clusters. Each cluster consists of a HIB (Host Interface Board), a CB (Control Board), and PBs (Processor Boards). DEC Alpha AXP 3000/x00 machines are used as the host computer, and HIBs are attached to the DEC TURBOChannel bus. For the connection between HIB and CB, we used a data transfer protocol which we call Hlink (HARP-link). It is a synchronous protocol with 32-bit data width. CB and PBs are connected by the custom bus with 96-bit data width and synchronous protocol with fixed latency, which we call HBus (HARP-Bus).

$\mathrm{PBs}$ calculate gravitational forces with parallel pipelines. A PB contains 47 HARP-Chips each of which has two virtual pipelines to calculate gravitational forces and operates on $32 \mathrm{MHz}$ clock cycle. One $\mathrm{PB}$ contains 94 virtual gravitational pipelines which operate on $16 \mathrm{MHz}$ clock cycle. CBs sum up the forces calculated on PBs and return the results to the host. HIBs translate the TURBOChannel protocol to Hlink protocol and vice versa.

\section{2. $\quad N$-body Simulation with the GRAPE-4 System}

The simplest $N$-body simulation with the GRAPE4 system proceeds in following order:

1) The host sends the information of $N$ particles to the memory on PBs where $N$ is the number of the particles in the system. We call this operation " $j$-particle write operation". For brevity, we call the particles of which data are stored in the memory of PB as " $j$-particles".

2 ) The host sends the information of $n_{\mathrm{vp}}$ particles to the pipelines on PBs. Here $n_{\mathrm{vp}}$ is the number of virtual pipelines on one $\mathrm{PB}$, which tipically equals to 94 . We call this operation " $i$ - particle write operation". We call the particles to be stored on the registers as " $i$-particles".

3) The pipelines on PBs calculate the forces for $n_{\mathrm{vp}} i$-particles.

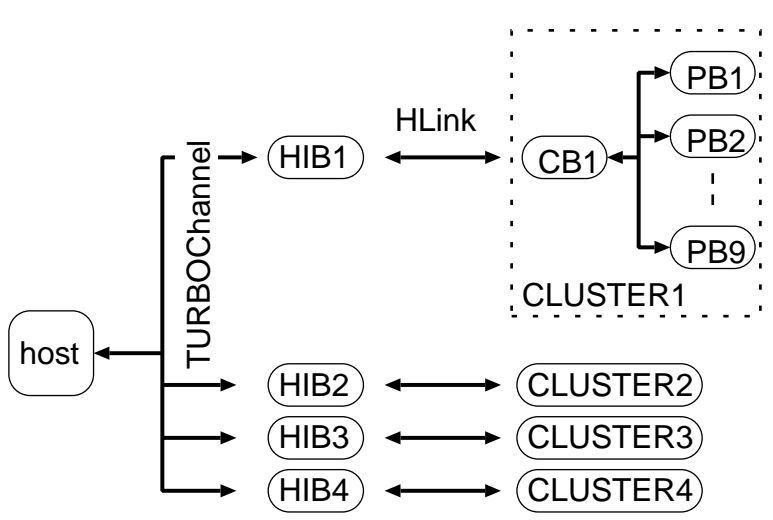

Fig. 1. - The GRAPE-4 System 
4) The host receives $n_{\mathrm{vp}}$ calculation results from pipelines on PBs. We call this operation "result read operation".

5) The procedures 2) to 4) are repeated until the calculation results for all $i$-particles are received.

6) The host updates the positions of all particles in the system.

\subsection{The Funciton of HIB}

In the following, we describe the function of HIB in some details, since its functionality is replicated in the new PCI version of HIB which will be described in the next section.

HIB communicates with the host in four different ways, namely programmed (PIO) write, PIO read, DMA read and DMA write. Here, programmed read/write means that the host computer initiates the transaction, while DMA means HIB initiates the transaction. PIO write is used by the host to send single-word commands to $\mathrm{CB}$ or $\mathrm{PB}$, and to set the HIB registers for DMA operation. PIO read is used only to read the status register of HIB. DMA read is used for data transfer to $\mathrm{CB} / \mathrm{PB}$ from the host, and DMA write is used for data transfer from $\mathrm{CB} / \mathrm{PB}$ to the host.

This rather complex implementation is quite different from that of the I/O interface of older GRAPE systems, which used PIO read/write operations on VME interface to perform all data transfer. We used DMA to achieve higher performance. In the case of the TURBOChannel bus on DEC Alpha AXP systems, the data transfer speed close to the theoretical maximum of $100 \mathrm{MB} / \mathrm{s}$ can be achieved with DMA, while the throughput of PIO read is around $10 \mathrm{MB} / \mathrm{s}$. However, the necessary bandwidth for GRAPE-4 is close to $100 \mathrm{MB} / \mathrm{s}$, and faster data transfer would further improve performance. Thus, the limited bandwidth of PIO operation on TURBOChannel was not satisfactory. The hardware to implement DMA operation on a TURBOChannel card was relatively simple, since TURBOChannel is an exceptionally simple $\mathrm{I} / \mathrm{O}$ bus. For example, the host CPU or any other device cannot interrupt a DMA operation, so there is no need to handle interrupt and restart of a DMA operation. There is no need for arbitration since each card slot has its dedicated signal lines for DMA request/grant. Of course, this rather simple protocol comes with several limitations. A single DMA operation must not transfer more than 128 words, and it should not go across the $2 \mathrm{~KB}$ address boundary.

We decided to design the interface between HIB and $\mathrm{CB}$ to be independent of the TURBOChannel protocol. The HIB hardware takes care of all the limitations of the TURBOChannel protocol. To hide the limitations of the TURBOChannel bus, HIB implements a bidirectional data buffer, which is large enough to hold all data to be moved between the host and $\mathrm{CB} / \mathrm{PB}$ by single request.

The data transfer in Hlink has three different modes. The first one is single-word handshaked transfer from HIB to CB, which is used to send commands. The second one is the burst transfer from HIB to CB, and the last one is the burst transfer from CB to HIB. The bursts can be interrupted by the sender, but the receiver must always accept the data. The way the transfer takes place and the length of the bursts are determined by the command sent from HIB to CB.

\section{The PCI-HIB}

In this section, we describe the PCI-HIB (PHIB for short) which replaces the TURBOChannel version of the HIB (THIB for short) described in the previous section. First we briefly overview the PCI bus, and compare it with other widely used standard I/O buses such as ISA, VME, SBus and the TURBOChannel. Then we describe the design principle and the hardware of the PHIB in detail.

\subsection{The PCI Standard}

The PCI interface was proposed by Intel in 1991 and standardized in 1992 as PCI version 1.0. Version 2.0 was defined in 1993 by PCI Special Interest Group(PCI Special Interest Group 1993) and the current standard is version 2.1. The PCI interface is a synchronous, high-performance I/O bus which has many advantages over other I/O buses. These advantages include

- Very high throughput, up to $533 \mathrm{MB} / \mathrm{s}$ in the case of $66 \mathrm{MHz}, 64$-bit transfer.

- Low manufacturing cost because of the use of the card-edge connector.

- Low power consumption because of the elimination of passive terminators. 
- Form factor similar to that of the ISA bus, which allows co-existence of PCI and ISA cards in one box.

- Sophisticated transaction protocols such as the support for the cache coherency.

- Support for automatic configuration which eliminates the need to set any jumper switches on board.

Partly because of these technical advantages and partly because of the fact that PCI is proposed by Intel, which is the largest manufacturer of microprocessors, PCI has quickly become a widely accepted standard. Table 1 summarizes the comparison between PCI and several other I/O specifications.

One problem with the PCI interface is that it is by far more complex compared to any other bus standards except Futurebus+. The support for the automatic configuration makes it necessary to implement rather complex logic for initial configuration process. Thus, even a simple slave interface board needs to implement rather complex function. A master interface board is still more complex, because the DMA transfer of PCI is interruptable.

\subsection{Design Principles of PHIB}

The goal for the design of the PHIB is quite simple. It needs to implement all the necessary functions available on the THIB. Even so, there are many considerations.

A rather important technical decision is whether to use the DMA transfer or not. In the case of the THIB, we had to use DMA since the performance of PIO read/write was unacceptably low. However, in the case of the PCI on workstations or PCs, the performance of PIO operation is not much different from that of the DMA transfer. For example, DEC Alphastation 5/600 offers the PIO write throughput of 119 $\mathrm{MB} / \mathrm{s}$ and read throughput of $56 \mathrm{MB} / \mathrm{s}$, according to the published document from DEC (Zurawski et al. 1995). PIO write performance in excess of $80 \mathrm{MB} / \mathrm{s}$ has been reported on a Pentium-based PC (Ichikawa and Shimada 1996).

The above numbers are still somewhat lower than what can be achieved with DMA. However, in most of recent machines, DMA operation needs additional data moving in the main memory which further degrades the effective transfer speed. The DMA operation can only move a block of the data in main memory, which must be physically in the main memory. This means that the data must be physically moved between the main memory and the cache. Unfortunately, the data transfer speed between the main memory and the cache is not much different from the speed of the I/O bus. Thus, a factor-of-two or more loss in performance is associated with the DMA operation.

Another problem with the DMA operation is that the software becomes much more complicated and error-prone. In order for an interface card to perform the DMA operation, the card needs to know the physical address of the memory to access. Since almost all operating systems now support the virtual memory, the user program knows only the virtual address of the data and has no knowledge about the physical memory. Of course the OS kernel keeps track of the mapping between the virtual address and physical address, but in order to access this information, we have to write software. Moreover, if a page fault occurs, the OS might change this mapping, or even put out a virtual page to the disk, without notifying the user process. If the OS has the support for realtime operations, it can prevent the specified memory region from being paged out, thereby preserving the mapping between the physical and virtual addresses. However, this functionality is not available in many popular UNIX systems, or rather awkward to use.

In the case of the PIO operation, the interface card need not know anything about the page mapping and the user process can simply read/write the virtual address assigned to the interface card, through usual load/store instruction, in other words, through assignment statement in any high level language. The main body of the software is portable among different platforms (Makino and Funato 1993).

For the present version of PHIB, therefore, we decided to use only the PIO operation. Thus, the data transfer which was performed using DMA in THIB is at present performed using PIO.

Even though we decided not to use the DMA operation, the implementation of the PCI interface is still rather complicated. Therefore we decided to use a general-purpose PCI interface chip, the PCI9060 from PLX technology Inc., as the interface to the PCI.

The PCI9060 chip integrates both the slave (for PIO) and master (DMA) functionalities in a single chip. In addition, it has all the necessary registers and logics for the automatic setup, thus eliminating any 
TABLE 1

COMPARISON BETWEen PCI AND OTHER I/O SPECIFICATIONS.

\begin{tabular}{llrrrrrr}
\hline \hline bus name & $\begin{array}{l}\text { synchronous/ } \\
\text { asynchronous }\end{array}$ & $\begin{array}{c}\text { clock } \\
\text { cycle } \\
(\mathrm{MHz})\end{array}$ & $\begin{array}{c}\text { bus } \\
\text { width } \\
\text { (bit) }\end{array}$ & $\begin{array}{c}\text { transfer } \\
\text { rate } \\
(\mathrm{MB} / \mathrm{s})\end{array}$ & $\begin{array}{c}\text { arbitration } \\
\text { type }\end{array}$ & $\begin{array}{c}\text { pin } \\
\text { count }\end{array}$ & $\begin{array}{c}\text { physical } \\
\text { dimemsion } \\
(\mathrm{mm})\end{array}$ \\
\hline ISA & synchronous & 8 & 16 & 8 & centralized & 88 & $107 \times 334$ \\
VME & asynchronous & 10 & 32 & 40 & centralized & 82 & $100 \times 160$ \\
NuBus & synchronous & 10 & 32 & 40 & centralized & 50 & $102 \times 327$ \\
TURBOChannel & synchronous & 25 & 32 & 100 & centralized & 44 & $117 \times 144$ \\
SBus & synchronous & 25 & 64 & 200 & centralized & 66 & $84 \times 142$ \\
PCI & synchronous & 66 & 64 & 533 & centralized & 102 & $107 \times 175$ \\
Futurebus+ & asynchronous & 100 & 128 & 1600 & centralized/distributed & 206 & $265 \times 297$ \\
\hline
\end{tabular}

need for designing logics specific to PCI. Its interface to the local bus is designed so that it can be directly connected to the CPU bus of the Intel i960 chips and act as both a DMA master and a PIO slave. In the present PHIB, the PCI9060 chip acts as a PIO slave to the host computer and as a DMA master to the local bus.

The PCI9060 interface chip can acts as the DMA master for both the PCI bus and i960 local bus simultaneously. Thus, the PHIB can perform the DMA operation to the PCI bus without any change in the design. This possibility allows us to design the PHIB with extra safety: If the actual performance of PIO is less than satisfactory, we can always implement the software for DMA to improve the performance.

\subsection{The Hardware Design}

Figure 2 shows the block diagram of PHIB. It consists of the PCI9060 chip, the Memory Controller chip (MC), and the dual-port memory unit. Figure 3 shows the photograph of the PHIB board.

The data transferred between the host and GRAPE are buffered by the dual-port memory unit. MC generates the addresses and other control signals for both ports of the memory, as well as the handshake signals to PCI9060 chip and the Hlink.

In THIB, we used the FIFO chips for the data buffer, which are much easier to use than the dualport memory. We do not have to provide the address for FIFO, and it can tell whether there are data to be sent or not. In addition, it is relatively easy to design the system in which the two ports of the FIFO chip operate on two independent clocks, since the flag logic implemented in the FIFO chip takes care of asynchronous two clocks.

In PHIB, however, we were forced to use the dualport memory. The reason is that the PCI interface need to support the read prefetch in order to allow the host to perform a burst read operation. In the case of a PCI burst read operation, the master can terminate the burst at any moment. Thus, the PCI9060 chip need to prefetch the data to supply, and when the burst is stopped, data already prefetched are discarded. Since some of the data read from the buffer will be discarded, we cannot use the FIFO as the data buffer. To read the same data more than once from an FIFO device is rather difficult. Therefore, we used dual-port memory chips. We used four IDT7099 chips from Integrated Device Technology, each of which has 8 -bit width and $4 \mathrm{k}$-word depth.

In the case of the data transfer from the host to $\mathrm{CB}$, the host first stores the data to the dual-port memory and then sets the register within $\mathrm{MC}$ to be the top address of the region with valid data. MC then sends out the new data to CB automatically. Figure 4 shows how the data transfer from host to $\mathrm{CB}$ proceeds.

In the case of the data transfer from CB to the host, when PHIB receives a data, it stores the received data to the dual-port memory and increments the address counter. The host computer tests if new data are ready or not by reading this counter, and if there are 


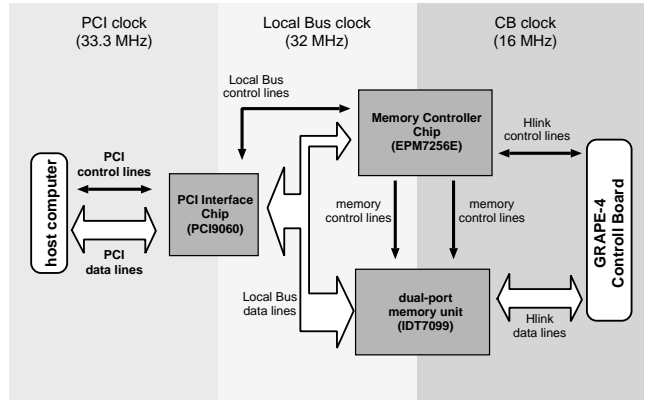

Fig. 2. - The block diagram of the PCI Host Interface Board
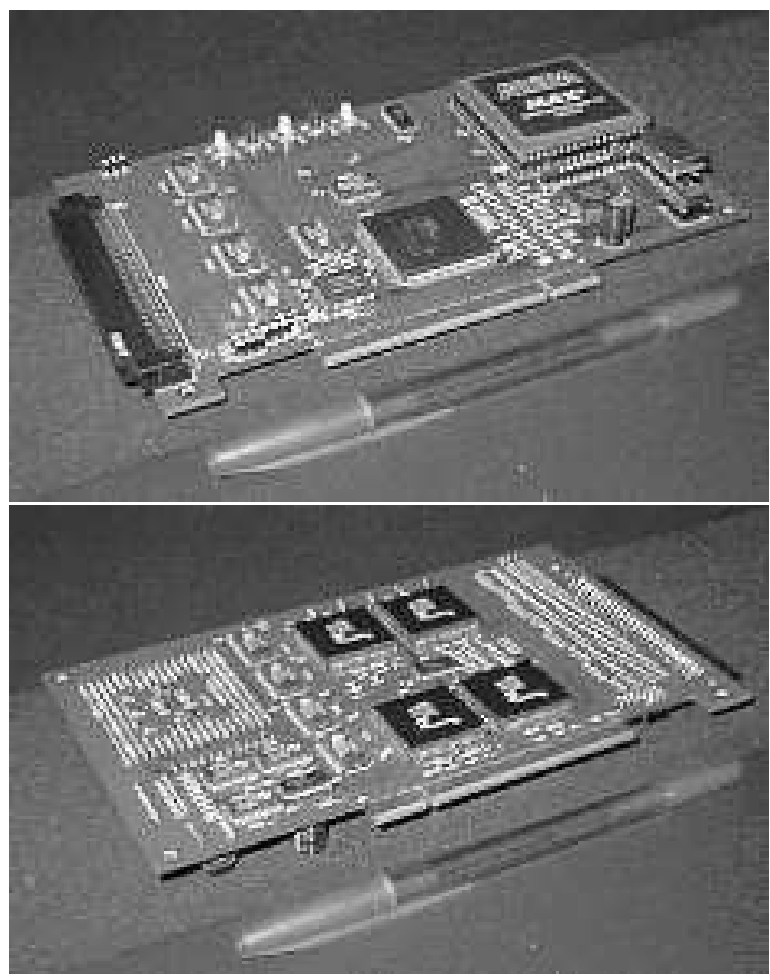

Fig. 3.- The PCI Host Interface Board. (a)The parts side. (b) The solder side.

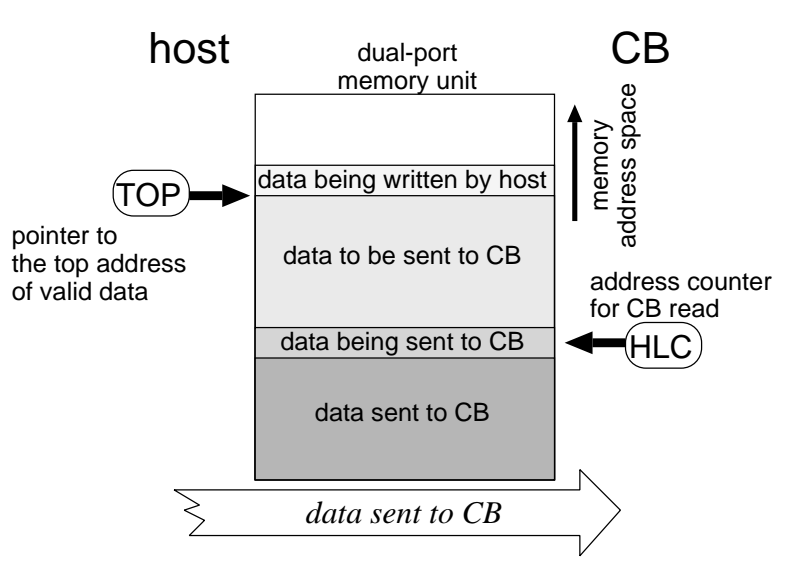

Fig. 4.- Data transfer from the host to CB. the HLC(Hlink-port address counter) and the TOP register in $\mathrm{MC}$ are used in this transfer. $\mathrm{CB}$ continues to read from the host computer until HLC catches up with TOP. The host modifies the TOP register to indicate $\mathrm{CB}$ that data to be transferred is ready.

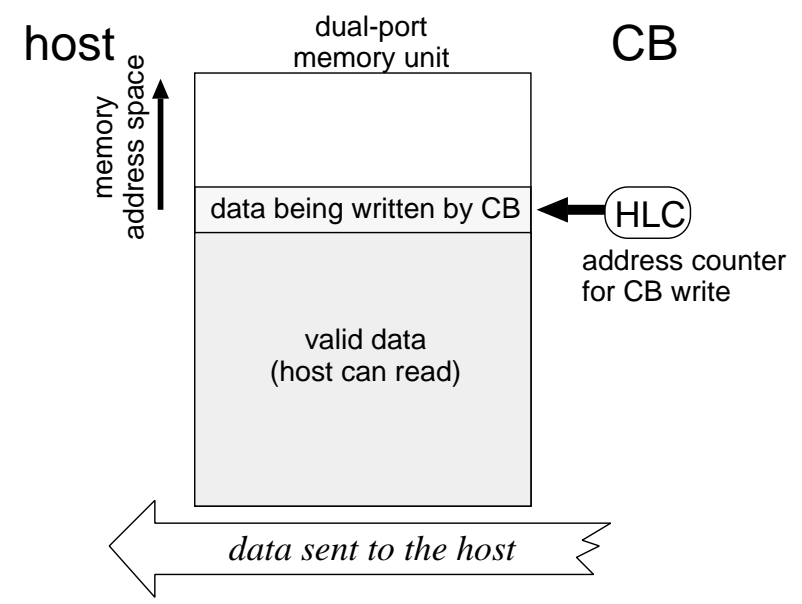

Fig. 5.- Data transfer from CB to the host. HLC register is used in this transfer. HLC points to the address of dual-port memory at which $\mathrm{CB}$ write data. 
new data it reads them in. Figure 5 shows how the data transfer proceeds.

The interface to Hlink operates on the clock signal from CB. The interface to PCI (part of the PCI9060 chip) operates on the PCI clock. The local bus of the PCI9060 operates on the clock independent of either of two external clocks. The speed of these three clocks are $16 \mathrm{MHz}, 33.3 \mathrm{MHz}$, and $32 \mathrm{MHz}$, respectively. Figure 2 shows which parts of the PHIB operate using which clock. PCI9060 accept the PCI and Local Bus clock. Part of MC and one port (Local Bus side) of the dual port memory operates on the LB clock. The remaining part of $\mathrm{MC}$ and the dual port memory operate on the clock from Hlink. MC unit is implemented in a single Altera EPM7256E complex PLD chip.

The data width of both PCI and Hlink are 32-bit. Thus the theoretical maximum of the transfer rate for these buses are $134 \mathrm{MB} / \mathrm{s}$ and $64 \mathrm{MB} / \mathrm{s}$, respectively.

\section{Performance}

In this section, we describe the performance of PHIB and compare it with that of THIB on real calculation. In section 4.1, we give the raw speed of the PCI interface itself, and discuss the dependence on the host. In section 4.2, we present the data transfer performance of library functions. In section 4.3 through 4.5 , we show the performance of the direct-summation equal-timestep algorithm, directsummation individual-timestep algorithm, and that of Barnes-Hut treecode, respectively.

\subsection{Raw Data Transfer Speed}

Table 2 shows the raw speed of the data transfer between the host and PHIB. Here, we simply measure the speed at which the host reads/writes a continuous region of the dual-port memory on PHIB. Alpha denotes an Alphastation 5-500/500 with 500MHz DEC 21164 processor and DEC 21172 PCI chipset. PC is an Intel P6 box from a Japanese company with a $200 \mathrm{MHz}$ Pentium Pro processor and Intel 440FX PCI chipset.

For the Alpha box, the performance is satisfactory, if not ideal. For the Intel box, however, the performance is rather low, in particular for the read operation. The difference of the speed of the write operation between these two hosts is not very large. The reason for the rather big difference in the speed of read operation is that the Alpha box performs the
PCI read burst for up to 32 bytes, while the particular Intel box we used performs only single-word (4 bytes) reads. The Alpha 21164 CPU can perform the load-merging, which combines consecutive load requests to the PCI bus to a single burst of up to 32 bytes. Thus, if the PCI device is fast enough, the data transfer speed of up to $56 \mathrm{MB} / \mathrm{s}$ can be achieved. On the other hand, the PCI interface chipset on the Intel box we used (Intel 440FX) does not have the capability of load-merging (or at least we could not figure out how to let that chip do the load merging. Intel documents give rather little information). At least one PCI chipset for Intel Pentium processor seems to perform load-merging (ALI M1448/1449 chipset). We hope similar chipsets to appear for Pentium Pro processors soon.

The read/write speed of $28 \mathrm{MB} / \mathrm{s}$ and $68 \mathrm{MB} / \mathrm{s}$ measured on the Alpha box are $50 \%$ and $60 \%$ of the maximum values in DEC document, respectively. These performance degradation is mainly because of the access latency of PCI9060 and MC. Taking these latencies into account, the actual throughput $P_{\text {raw }}$ can be approximated as

$$
P_{\text {raw }}=P_{\text {max }} \frac{B}{B+L_{9060}+L_{\mathrm{mc}}+L_{\mathrm{host}}}
$$

where $P_{\max }=133 \mathrm{MB} / \mathrm{s}$ is the maximum data transfer speed of PCI and $B$ is the length of the PCI burst in clock counts. On the Alpha box we used, $B=8$. The parameters $L_{9060}, L_{\mathrm{mc}}$, and $L_{\text {host }}$ are the access latencies of PCI9060, MC, and the host, in unit of PCI clock. The values for these parapeters are shown in table 3 both in unit of ns and of PCI clock. Equiation (1) gives the read/write speed of $30 \mathrm{MB} / \mathrm{s}$ and $76 \mathrm{MB} / \mathrm{s}$. These values agree well with the measured performance.

\subsection{Data Transfer Speed of Library Func- tions}

In this section we present the data transfer performance of library functions.

As described in section 2.2, the main body of data transfer in actual simulation is divided into three parts: $j$-particle write operation, $i$-particle write operation, and result read operation. Figure 6 shows the measured speed of data transfer for these three operations as a function of the number of particles. The host computer we used was a DEC AlphaStation 5-500/500 with 128MB memory (AS500). 
TABLE 2

PHIB PERFORMANCE

\begin{tabular}{llc}
\hline \hline \multicolumn{1}{c}{ host } & Write & Read \\
\hline AS500(21164/500MHz) & $68 \mathrm{MB} / \mathrm{s}$ & $28 \mathrm{MB} / \mathrm{s}$ \\
PC(P6/200MHz) & $58 \mathrm{MB} / \mathrm{s}$ & $10 \mathrm{MB} / \mathrm{s}$ \\
\hline
\end{tabular}

TABLE 3

AcCess latency of PCI9060, MC, and the host.

\begin{tabular}{lcc}
\hline \hline Latency source & Write & Read \\
\hline PCI9060 & $60 \mathrm{~ns}(2$ clocks) & $390 \mathrm{~ns}(13$ clocks $)$ \\
MC & $90 \mathrm{~ns}(3$ clocks $)$ & $120 \mathrm{~ns}(4$ clocks $)$ \\
host & $30 \mathrm{~ns}(1$ clocks $)$ & $330 \mathrm{~ns}(11$ clocks $)$ \\
\hline
\end{tabular}

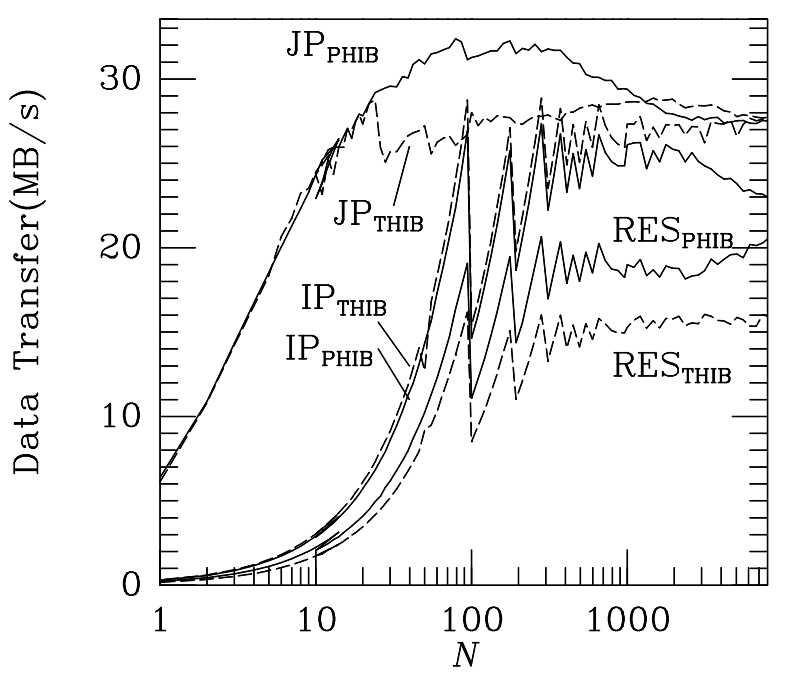

Fig. 6. - The measured data transfer speed of library functions plotted as a function of the number of particles. The curves labeled IP, JP, and RES indicate the performance of $i$-particles write operation, $j$-particles write operation, and calculation result read operation, respectively. The performance of the PHIB system are shown in solid curves, and that of the THIB system are shown in dashed curves.
The curves for $j$-particles write operation is smooth for both of PHIB and THIB, while that for $i$-particles write and result read operations show discontinuous drop at $N=n_{\mathrm{vp}}, 2 n_{\mathrm{vp}}, 3 n_{\mathrm{vp}} \ldots$, where $n_{\mathrm{vp}}(=94)$ is the number of virtual pipelines on $\mathrm{PB}$. This is because the data transfer in those operations are performed on block of $n_{\mathrm{vp}}$ particles. For $N$ which is not an exact integer multiple of $n_{\mathrm{vp}}$, part of the data transferred in $i$-particle write and result read operations are not used. Thus for $N \ll n_{\mathrm{vp}}$, the speed of these two operations can be very low. To be more precise, the time to send $n j$-particles can be approximated as

$$
T_{\mathrm{j}}=C_{1}+C_{2} n,
$$

where $C_{1}=8.5 \times 10^{-6} \mathrm{sec}$ and $C_{2}=2.5 \times 10^{-6} \mathrm{sec}$ while that for $i$-particle write and result read are

$$
T_{\mathrm{i}}=C_{\mathrm{i}}\left[\frac{n+n_{\mathrm{vp}}-1}{n_{\mathrm{vp}}}\right],
$$

and

$$
T_{\mathrm{r}}=C_{\mathrm{r}}\left[\frac{n+n_{\mathrm{vp}}-1}{n_{\mathrm{vp}}}\right],
$$

where $C_{\mathrm{i}}=1.4 \times 10^{-4} \mathrm{sec}$ and $C_{\mathrm{r}}=1.9 \times 10^{-4} \mathrm{sec}$. Here, $[x]$ denotes the maximum integer which does not exceed $x$. 
The peak performance of the data transfer through library functions is somewhat lower than that of the raw data transfer. For the read operation, this low performance is mainly due to the cost of data transfer within the main memory of the host. After the data read from PHIB are stored in a continuous memory block, each data must be moved to appropriate address given by the user of the library. To see the cost of data transfer within the main memory, we measured the performance of the read operation without memory copy and got the speed of $\sim 25 \mathrm{MB} / \mathrm{s}$. This result can explain the difference between the performance of the raw data read and that of the result read operation. For the write operations, the major reason for the performance reduction is that the host has to fetch data from the main memory. In actual simulation, these data are scattered in large region of the main memory $(\geq 10 \mathrm{MB})$. In addition, the structure of the data to be sent to PHIB and that of the data given by the user are different. Thus the write operations have to fetch data from wide and noncontiguous range of the main memory. To see the cost of data fetch from main memory, we measured the write speed in two different ways. We used data which occupy more than 50MB of the main memory. In the measurement described in section 4.1, we used data in the register of CPU. In the first test, we sent them to PHIB in contiguous order. In this measurement, the write speed was reduced to $\sim 50 \mathrm{MB} / \mathrm{s}$. In the second, we used the same data but sent them in non-contiguous order. In this case, the write speed was reduced to $\sim 30 \mathrm{MB} / \mathrm{s}$. These results are consistent with the performance of the write operations.

Compared to the transfer speed of these operations, the speed of Hlink is high enough $(64 \mathrm{MB} / \mathrm{s})$ and does not limit the performance of the PHIB system.

\subsection{Direct Summation Code, Equal-timestep}

We performed test runs with an equal-timestep code. As the initial conditions, we used a Plummer model. We changed the number of particles $N$ from 1024 to 262144 and measured the speed of calculation with one PB. The system of units is chosen so that the total mass of the system $M$ and the gravitational constant $G$ are both unity. The total energy of the system $E$ is $-1 / 4$ (Heggie and Mathieu 1986). The mass of all particles are $m=1 / N$. The softening parameter is $N^{-1 / 3}$, where $N$ is the number of particles. The host computer was an AS500. We performed the same run with the THIB and Alpha AXP 3000/700 (225MHz 21064A processor with 64MB memory, hereafter AA3000). 
Figure 7a shows the calculation speed of GRAPE-4 in Gflops and figure 7b shows the average CPU time per one timestep, for both PHIB and THIB.

The time to integrate the system of $N$ particles for one timestep is estimated as follows:

$$
T_{\text {eq }}=T_{\text {host } 1}+T_{\text {grape1 }}+T_{\text {comm1 }},
$$

where $T_{\text {host } 1}, T_{\text {grape } 1}$, and $T_{\text {comm1 } 1}$ are the time spent on host, the time spent on GRAPE-4, and the time spent for data transfer between the host and GRAPE4 , respectively. The three terms in the right-hand side of equation (5) are expressed as

$$
\begin{aligned}
T_{\text {host1 }} & =N t_{\text {host1 }}, \\
T_{\text {grape1 }} & =g_{1} \frac{3 N^{2} t_{\text {pipe }}}{n_{\mathrm{vp}}}, \\
T_{\text {comm1 }} & =N\left(19 t_{\mathrm{jp}}+10 g_{1} r t_{\mathrm{ip}}+10 g_{1} r t_{\mathrm{res}}\right) .
\end{aligned}
$$

Here, $t_{\text {host } 1}$ in equation (6) is the time for the host computer to integrate one particle for one timestep. In equation (7), $n_{\mathrm{vp}}$ is the number of virtual pipelines on $\mathrm{PB}$, and $t_{\text {pipe }}$ is the cycle time of PB. For AS500, $t_{\text {host } 1}=0.5 \times 10^{-6} \mathrm{sec}$ and for AA3000, $t_{\text {host } 1}=1.0$ $\times 10^{-6}$ sec. For the system we used, $n_{\mathrm{vp}}=94$. The clock cycle of $\mathrm{PB}$ is $16 \mathrm{MHz}$, which corresponds to $t_{\text {pipe }}=6.25 \times 10^{-8}$ sec. The parameter $g_{1}$ is the loss of parallel efficiency of multiple pipelines, which is estimated as

$$
g_{1}=\left[\frac{N+n_{\mathrm{vp}}-1}{n_{\mathrm{vp}}}\right] \frac{n_{\mathrm{vp}}}{N} .
$$

In equation (8), $t_{\mathrm{jp}}, t_{\mathrm{ip}}$, and $t_{\mathrm{res}}$ are the time to transfer one word ( 4 bytes) in $j$-particle write operation, $i$-particle write operation, and result read operation, respectively. For these parameters, we use the values measured at $N=10^{4}$ (See figure 6). When $N$ is larger than 43690 , which is the size of the $j$-particle memory of $\mathrm{PB}$, we have to divide $j$-particles to groups with the number of particles not exceeding 43690. We can calculate the total forces by summing up the forces from each groups. The parameter $r$ denotes the number of such groups, which is given by

$$
r=\left[\frac{N-1}{43690}+1\right] .
$$

Figure $7 \mathrm{c}$ and $7 \mathrm{~d}$ shows the theoretical estimate of fraction of the time spent on the host calculation, data transfer, and calculation on GRAPE for PHIB and THIB, respectively.
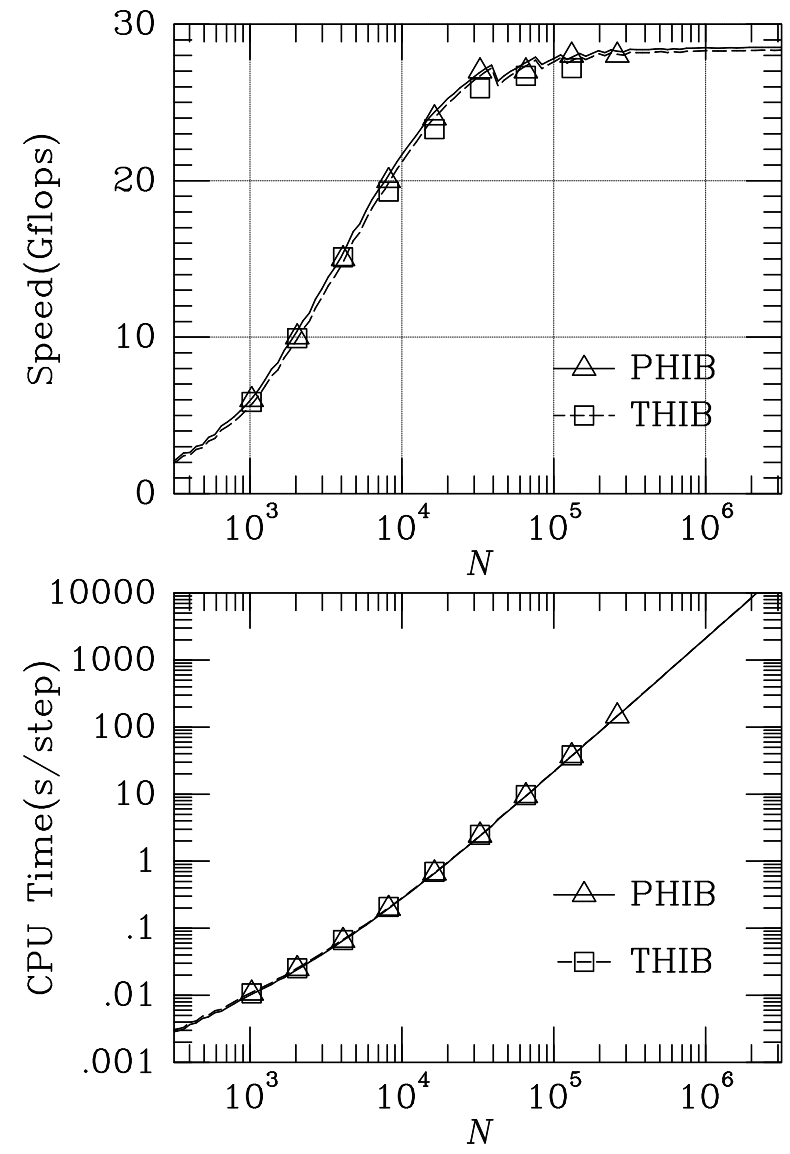

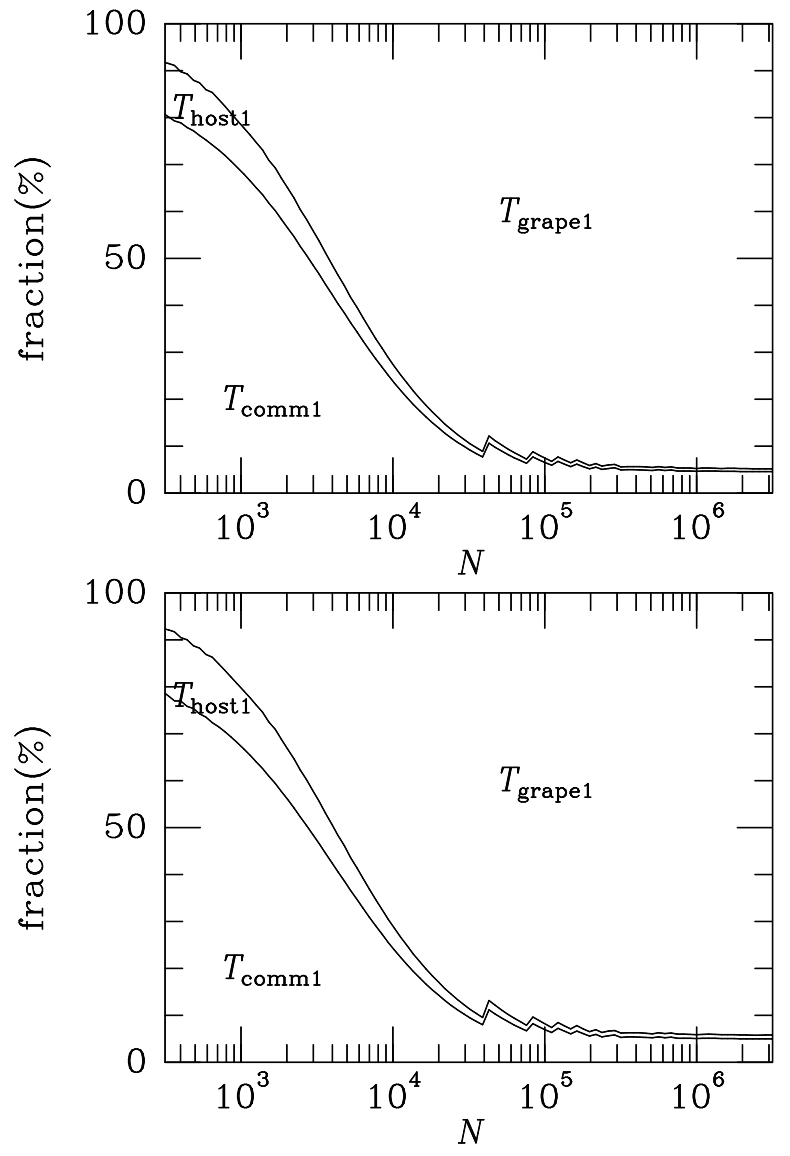

Fig. 7.- (a) The calculation speed and (b) CPU time per one timestep of GRAPE-4 for equal-timestep algorithm, plotted as a function of the number of particles in the system. Solid and dashed curves represent the theoretical estimate for PHIB and THIB, respectively. Triangles and Squares represent the measured performance of PHIB and THIB, respectively. (c) The fraction of the time spent on the host calculation, data transfer, and calculation on GRAPE for the PHIB system, plotted as a function of the number of particles in the system. (d) Same as figure 7c, but for the THIB system.

In figures $7 \mathrm{a}$ and $7 \mathrm{~b}$, the calculation time of the PHIB system is only $5 \%$ faster than that of the THIB system. This is because the host calculation time in this algorithm is small $(\sim 10 \%)$. The communication performance with PHIB is almost same as that with THIB and the difference of these two system are mainly exist in the performance of the hosts. Since the calculation time spent on the host is small, the performance of the two systems are not so much different.

\subsection{Direct Summation Code, Individual-timestep}

We performed test runs with an individual-timestep code. The initial condition and hardware configuration are the same as the ones used in the test run of equal-timestep code described in section 4.3, except that the softening parameter is $2 / N$. We changed the number of particles from 1024 to 32768 , and measured the speed of calculation.

Figure 8a shows the calculation speed of GRAPE-4 in Gflops and figure 8b shows the average CPU time to integrate one particle for one timestep, for both PHIB and THIB.

The time to integrate one particle for one timestep is estimated as follows:

$$
T_{\text {idt }}=T_{\text {host } 2}+T_{\text {grape } 2}+T_{\text {comm } 2},
$$

where $T_{\text {host2 }}, T_{\text {grape2 }}$, and $T_{\text {comm2 }}$ are the time spent on host, the time spent on GRAPE-4, and the time spent for data transfer between the host and GRAPE4 , respectively. In equation (11), the term $T_{\text {host2 }}$ is estimated as $1.3 \times 10^{-5} \mathrm{sec}$ for AS500 and $2.5 \times$ $10^{-5}$ sec for AA3000.

The terms $T_{\text {grape2 }}$ and $T_{\text {comm2 }}$ are expressed as

$$
\begin{aligned}
T_{\text {grape2 }} & =g_{2} \frac{3 N t_{\text {pipe }}}{n_{\mathrm{vp}}} \\
T_{\text {comm2 }} & =\left(38 t_{\mathrm{jp}}+10 g_{2} t_{\mathrm{ip}}+10 g_{2} t_{\mathrm{res}}\right) .
\end{aligned}
$$

Here, the parameter $g_{2}$ in equation (12) is the loss of parallel efficiency of multiple pipelines, which is estimated as

$$
g_{2}=\left[\frac{n_{\mathrm{s}}+n_{\mathrm{vp}}-1}{n_{\mathrm{vp}}}\right] \frac{n_{\mathrm{vp}}}{n_{\mathrm{s}}} .
$$

The parameter $n_{\mathrm{s}}$ is the average number of particles to share the same time. For this parameter, we used experimental result (Makino et al. 1997)

$$
n_{\mathrm{s}}=1.6 N^{1 / 2} \text {. }
$$




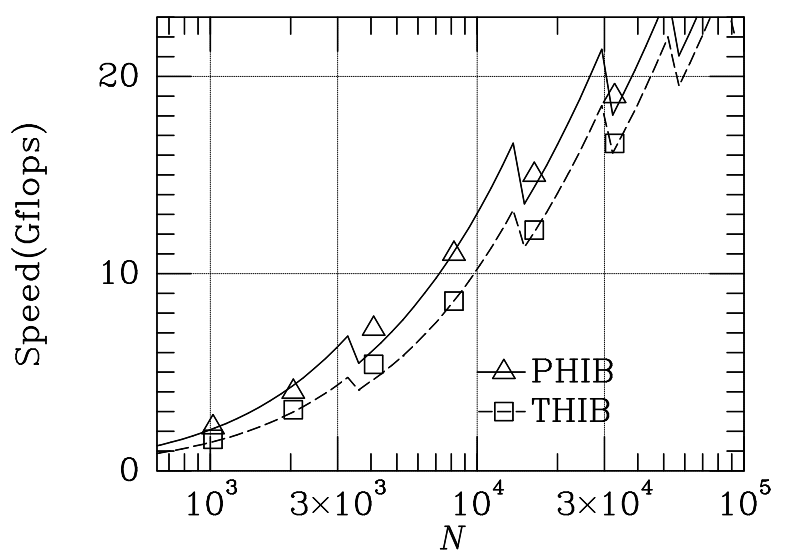

In the case of the individual-timestep algorithm, the PHIB system is 10-30\% faster than the THIB system. This is because the the host calculation term $T_{\text {host2 }}$ in equation (11) are comparable to $T_{\text {grape2 } 2}$ and $T_{\text {comm2 }}$ (See figure $8 \mathrm{c}$ and $8 \mathrm{~d}$ ). The reduction of the host calculation time with this algorithm is more significant than that with the equal-timestep algorithm.

\subsection{Barnes-Hut Treecode}

We performed test runs with a Barnes-Hut treecode modified for GRAPE (Makino 1991a). The initial condition and the hardware configuration are the same as the ones used in the test run of equal-timestep code described in section 4.3. The opening angle for cells of treecode, $\theta$, is 0.75 , and the critical particle number for Barnes' vectorization scheme, $n_{\text {crit }}$, is 4000. Strictly speaking, $n_{\text {crit }}$ depends on $N$ and must be optimized for each value of $N$ (Barnes 1990). However, the dependency of $n_{\text {crit }}$ on $N$ is weak, and we found that $n_{\text {crit }}=4000$ is close to optimum for the entire range of $N$ we used. We changed the number of particles from 16384 to 1048576 , and measured the speed of calculation.

Figure 9a shows the average CPU time per one timestep, for both PHIB and THIB. Figure 9b shows the CPU time per step per particle. On average, the PHIB system is about $40 \%$ faster than the THIB system.

The calculation time for one timestep is estimated as follows:

$$
T_{\text {tree }}=T_{\text {host } 3}+T_{\text {grape3 }}+T_{\text {comm } 3},
$$

where $T_{\text {host3 }}, T_{\text {grape3 }}$, and $T_{\text {comm } 3}$ are the time spent on host, the time spent on GRAPE-4, and the time spent for data transfer between the host and GRAPE4 , respectively. These terms are expressed as

$$
\begin{aligned}
T_{\text {host3 }}= & N t_{\text {int }}+\left(N \log _{10} N\right) t_{\text {const }} \\
& +\frac{N n_{\text {terms }}}{n_{\mathrm{g}}} t_{\text {list }}, \\
T_{\text {grape3 }}= & g_{3} \frac{3 N n_{\text {terms }} t_{\text {pipe }}}{n_{\mathrm{vp}}}, \\
T_{\text {comm3 }}= & N\left(19 \frac{n_{\text {terms }}}{n_{\mathrm{g}}} t_{\text {jp }}+\right. \\
& \left.10 g_{3} t_{\text {ip }}+10 g_{3} t_{\text {res }}\right) .
\end{aligned}
$$

In equation (17), $t_{\text {int }}$ is the time for the host computer to integrate one particle for one timestep, $t_{\text {const }}$ is the time to construct the tree structure, and $t_{\text {list }}$ is the

Fig. 8.- Same as figure 7 but for the individualtimestep algorithm. 

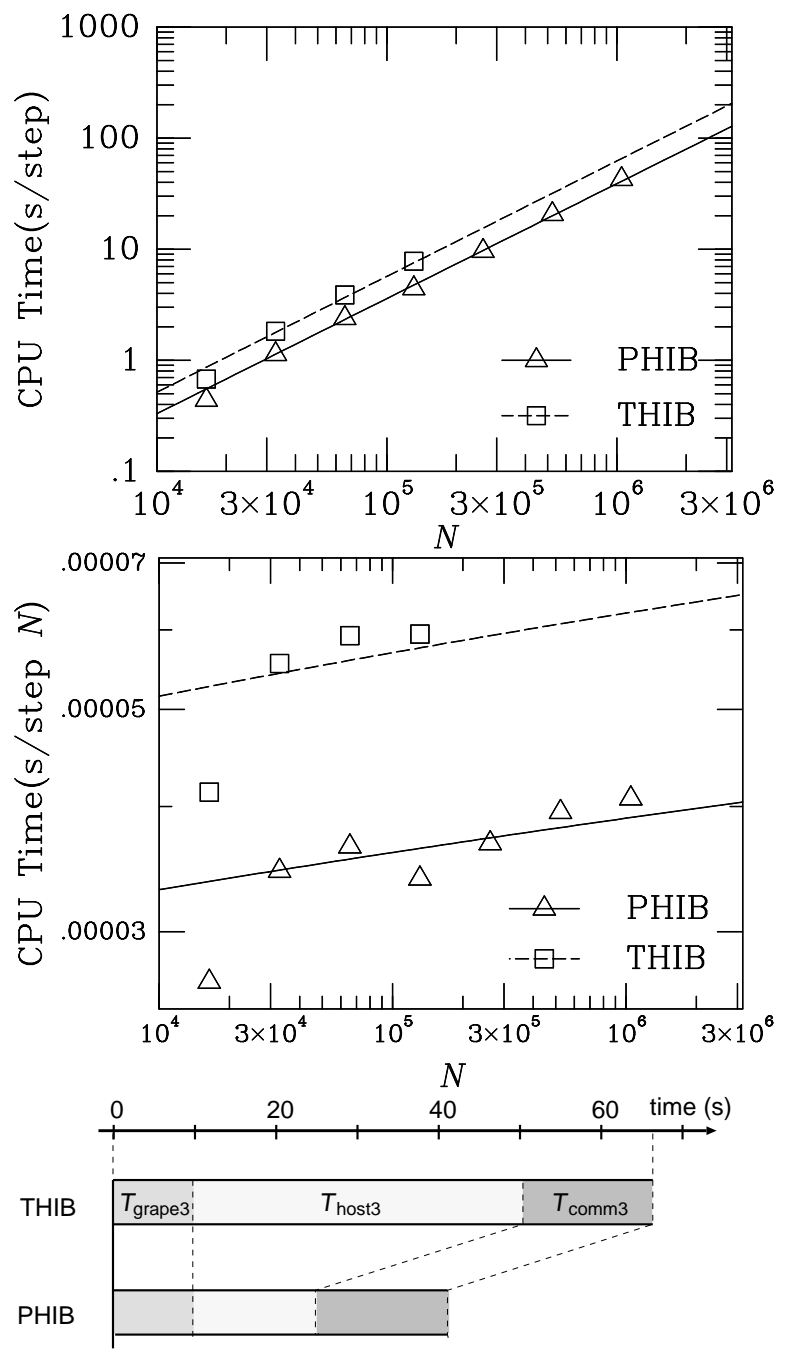

Fig. 9.- (a)Same as figure 7b, but for the BarnesHut treecode. (b)CPU time per one timestep per one particle for the Barnes-Hut treecode. The meaning of curves and symbols are the same as those in figure 9a. (c)Theoretical estimate of CPU time per one timestep spent on GRAPE, the host calculation, and data transfer for both PHIB and THIB. The number of particles is 1048576 .

time to create the interaction lists (Fukushige et al. 1991). These values are estimated to be $1.9 \times 10^{-6}$ sec, $1.6 \times 10^{-6} \mathrm{sec}$, and $0.8 \times 10^{-6} \mathrm{sec}$ for AS500 and $3.6 \times 10^{-6} \mathrm{sec}, 3.5 \times 10^{-6} \mathrm{sec}$, and $3.3 \times 10^{-6} \mathrm{sec}$ for AA3000, respectively. According to Makino(1991a), the average length of the interaction list, $n_{\text {terms }}$, and the number of particles in the group, $n_{\mathrm{g}}$, are estimated as

$$
\begin{aligned}
n_{\mathrm{g}} \simeq & n_{\text {crit }} / 4, \\
n_{\text {terms }} \simeq & n_{\mathrm{g}}+14 n_{\mathrm{g}}^{2 / 3}+84 n_{\mathrm{g}}^{1 / 3}+56 \log _{8} n_{\mathrm{g}} \\
& -31 \theta^{-3} \log _{10} n_{\mathrm{g}}-72 \\
& +10^{2} \theta^{-3} \log _{8} 10 \frac{N \theta^{3}}{23} .
\end{aligned}
$$

In equation (18), $g_{3}$ is the loss of parallel efficiency of multiple pipelines, which is estimated as

$$
g_{3}=\left[\frac{n_{\mathrm{g}}+n_{\mathrm{vp}}-1}{n_{\mathrm{vp}}}\right] \frac{n_{\mathrm{vp}}}{n_{\mathrm{g}}} .
$$

\section{Summary and Discussion}

In this paper, we presented the design and performance of PHIB, the PCI interface for the GRAPE-4 special-purpose computer. It allows us to use a wide variety of host computers, from Intel-based PCs to vector supercomputers.

Another important implication of PHIB is that we can use it as a generic host interface for our future projects. The protocol used in Hlink is quite simple and does not require sophisticated implementation, either in logical or electrical designs, and yet it can achieve the data transfer speed up to $100 \mathrm{MB} / \mathrm{s}$, with extremely small latency (the latency of a PIO read operation is less than $500 \mathrm{~ns}$ ). We plan to use PHIB

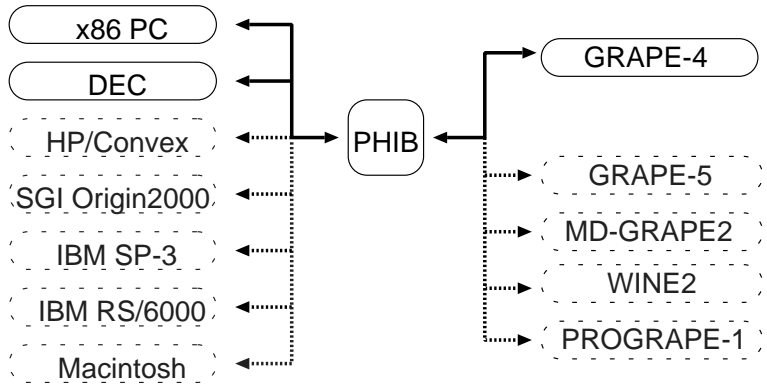

Fig. 10.- Future plan for PHIB. PHIB can be used to connect various host computers to GRAPE systems to be developed in future. 
as the interface to new systems we will develop in the next few years (See figure 10).

We thank Evangelie Athanassoulas for providing the host computer of GRAPE-4 system in performance measuring. A.K. would like to thank Evangelie Athanassoulas for her kind hospitality during his stay at Marseilles. This work was supported by Grant-inAid for Specially Promoted Reserch (04102002) of the ministry of Education, Science, Spotrts and Culture, Japan, and by National Science Foundation under an Advanced Scientific Computing grant ASC-9612029.

\section{REFERENCES}

Barnes J. E., Hut P. 1986, Nature 324, 446

Barnes J. E. 1990, J. Comp. Phys. 87, 161

Brieu P. P., Summers F. J., Ostriker J. P. 1994, ApJ 453, 566

Zurawski J. H., Murray J. E., Lemmon P. J. 1995, DEC Technical Journal Vol7, No.1, 89

Fukushige T., Ito T., Makino J., Ebisuzaki T., Sugimoto D. 1991, PASJ 43, 841

Heggie D. C., Mathieu R. D. 1986, in The Use of Supercomputers in Stellar Dynamics, ed S. McMillan, P. Hut (Springer, New York) p233

Ichikawa S., Shimada T. 1996, Technical Report of IEICE 85, 159 (in Japanese)

Makino J. 1991a, PASJ 43, 621

Makino J. 1991b, PASJ 43, 859

Makino J., Funato Y. 1993, PASJ 45, 279

Makino J., Taiji M. 1995, Proceedings of Supercomputing '95 (CD-ROM)

Makino J., Taiji M., Ebisuzaki T., Sugimoto D. 1997, ApJ 480, 432

Sugimoto D., Chikada Y, Makino J., Ito T., Ebisuzaki T., Umemura M. 1990, Nature 345, 33

Taiji M., Makino J., Fukushige T., Ebisuzaki T., Sugimoto D. 1996, in IAU Symposium 174 Dynamical Evolution of Star Clusters, ed J. Makino, P. Hut (Kluwer Academic Press, Dordrecht) p141

PCI Special Interest Group 1993, PCI Local Bus Specification Rev. 2.0 (PCI Special Interest Group, Hillsboro)

This 2-column preprint was prepared with the AAS IATEX

macros v4.0. 